\title{
Generalized optical theorem in coherent scattering
}

\author{
Ming Chiang Li \\ Department of Physics, Virginia Polytechnic Institute and State University, Blacksburg, Virginia 24061
}

(Received 14 October 1974)

It is shown that the generalized optical theorem in quantum mechanics is a useful theorem in the scattering of two coherent beams.

In a previous paper, ${ }^{1}$ the scattering of two coherent beams was studied. It was shown that, through the scattering experiment of two coherent beams, one is able to measure the imaginary part of the scattering amplitude at any angle in terms of coherent and conventional total cross sections. However, the discussion was confined to scattering by spherical and spheroidal potentials. It has been realized only recently that the conclusion is also true for scattering by any nonspherical and complex potential which possesses space-inversion symmetry. It is the intention of this comment to prove such a generalization.

In the coherent-scattering process, the wave function satisfies the Schrödinger equation

$$
-\left(\hbar^{2} / 2 m\right) \nabla^{2} \Psi(\overrightarrow{\mathrm{r}})+V(\overrightarrow{\mathrm{r}}) \Psi(\overrightarrow{\mathrm{r}})=E \Psi(\overrightarrow{\mathrm{r}})
$$

and the asymptotic condition

$$
\Psi(\overrightarrow{\mathrm{r}}) \underset{r \rightarrow \infty}{\longrightarrow} e^{i \overrightarrow{\mathrm{k}}_{1} \cdot \overrightarrow{\mathrm{r}}}+a e^{i \overrightarrow{\mathrm{k}}_{2} \cdot \overrightarrow{\mathrm{r}}}+F\left(\overrightarrow{\mathrm{k}}_{1}, \overrightarrow{\mathrm{k}}_{2} ; \overrightarrow{\mathrm{n}}\right) e^{i k r} / r .
$$

The potential $V(\overrightarrow{\mathrm{r}})$ is complex and has the form

$$
V(\overrightarrow{\mathbf{r}})=V_{R}(\overrightarrow{\mathbf{r}})-i V_{I}(\overrightarrow{\mathrm{r}}) \text {, }
$$

where $V_{R}(\overrightarrow{\mathrm{r}})$ and $V_{I}(\overrightarrow{\mathrm{r}})$ are real potentials and $V_{I}(\vec{r}) \geqslant 0$. The coherent incident beams have momenta $\overrightarrow{\mathrm{k}}_{1}$ and $\overrightarrow{\mathrm{k}}_{2}$ and energy $E$. Then

$$
\overrightarrow{\mathrm{k}}_{1}^{2}=\overrightarrow{\mathrm{k}}_{2}^{2}=\overrightarrow{\mathrm{k}}^{2}=2 m E / \hbar^{2},
$$

where $m$ is the mass of particles in the beams. In Eq. (2) $a$ is the relative phase and amplitude of these two incident beams and $F\left(\overrightarrow{\mathrm{k}}_{1}, \overrightarrow{\mathrm{k}}_{2} ; \overrightarrow{\mathrm{n}}\right)$ is the coherent-scattering amplitude in the direction $\vec{n}$.

From Eq. (1) it is easy to obtain the equation

$$
\begin{aligned}
-(\hbar / 2 i m)\left[\Psi *(\overrightarrow{\mathrm{r}}) \nabla^{2} \Psi(\overrightarrow{\mathrm{r}})\right. & \left.-\Psi(\overrightarrow{\mathrm{r}}) \nabla^{2} \Psi *(\overrightarrow{\mathrm{r}})\right] \\
& =(2 / \hbar) V_{I}(\overrightarrow{\mathrm{r}}) \Psi *(\overrightarrow{\mathrm{r}}) \Psi(\overrightarrow{\mathrm{r}}) .
\end{aligned}
$$

By carrying out the integration over a large spherical volume of radius $R$ and using Green's theorem one arrives at the equation

$$
\begin{aligned}
-\frac{\hbar}{2 i m} R^{2} \int\left(\Psi *(\overrightarrow{\mathrm{r}}) \frac{\partial}{\partial r}\right. & \left.\Psi(\overrightarrow{\mathrm{r}})-\Psi(\overrightarrow{\mathrm{r}}) \frac{\partial}{\partial r} \Psi *(\overrightarrow{\mathrm{r}})\right) d \Omega_{\overrightarrow{\mathrm{n}}} \\
& =\frac{2}{\hbar} \int_{R} V_{I}(\overrightarrow{\mathrm{r}}) \Psi *(\overrightarrow{\mathrm{r}}) \Psi(\overrightarrow{\mathrm{r}}) d \overrightarrow{\mathrm{r}}
\end{aligned}
$$

where $d \Omega_{\overrightarrow{\mathrm{n}}}$ and $d \overrightarrow{\mathrm{r}}$ are the solid angle and volume elements, respectively. The left-hand side of Eq. (6) denotes the flux of particles removed by the imaginary part $V_{I}(\overrightarrow{\mathrm{r}})$ of the complex potential. The right-hand side is usually expressed in terms of the coherent absorption cross section ${ }^{2}$ :

$$
\Sigma_{\mathrm{abs}}\left(\overrightarrow{\mathrm{k}}_{1}, \overrightarrow{\mathrm{k}}_{2}\right)=\left(2 m / \hbar^{2} k\right) \int_{R} V_{I}(\overrightarrow{\mathrm{r}}) \Psi *(\overrightarrow{\mathrm{r}}) \Psi(\overrightarrow{\mathrm{r}}) d \overrightarrow{\mathrm{r}} .
$$

The coherent-scattering amplitude can be written as the sum of the conventional-scattering amplitudes $f\left(\overrightarrow{\mathrm{k}}_{1} ; \overrightarrow{\mathrm{n}}\right)$ and $f\left(\overrightarrow{\mathrm{k}}_{2} ; \overrightarrow{\mathrm{n}}\right)$,

$$
F\left(\overrightarrow{\mathrm{k}}_{1}, \overrightarrow{\mathrm{k}}_{2} ; \overrightarrow{\mathrm{n}}\right)=f\left(\overrightarrow{\mathrm{k}}_{1} ; \overrightarrow{\mathrm{n}}\right)+a f\left(\overrightarrow{\mathrm{k}}_{2} ; \overrightarrow{\mathrm{n}}\right) .
$$

With the help of Eqs. (2) and (8) one can perform the angular integration in Eq. (6). The resulting equation has the form

$$
\begin{aligned}
\Sigma_{\mathrm{abs}}\left(\overrightarrow{\mathrm{k}}_{1}, \overrightarrow{\mathrm{k}}_{2}\right)= & -\Sigma_{e}\left(\overrightarrow{\mathrm{k}}_{1}, \overrightarrow{\mathrm{k}}_{2}\right)+\frac{4 \pi}{k\left(1+|a|^{2}\right)} \\
& \times\left[\operatorname{Im} f\left(\overrightarrow{\mathrm{k}}_{1} ; \overrightarrow{\mathrm{n}}_{1}\right)+|a|^{2} \operatorname{Im} f\left(\overrightarrow{\mathrm{k}}_{2} ; \overrightarrow{\mathrm{n}}_{2}\right)\right] \\
& +\frac{4 \pi}{k\left(1+|a|^{2}\right.} \operatorname{Im}\left[a f\left(\overrightarrow{\mathrm{k}}_{1} ; \overrightarrow{\mathrm{n}}_{2}\right)+a^{*} f\left(\overrightarrow{\mathrm{k}}_{2} ; \overrightarrow{\mathrm{n}}_{1}\right)\right],
\end{aligned}
$$

where

$$
\overrightarrow{\mathrm{n}}_{i}=\overrightarrow{\mathrm{k}}_{i} / k \text { for } i=1,2 \text {. }
$$

It has been assumed in Eq. (9) that $\overrightarrow{\mathrm{n}}_{1} \neq \overrightarrow{\mathrm{n}}_{2} ; \psi(\overrightarrow{\mathrm{r}})$ in Eq. (2) is then normalized by the factor $\left(1+|a|^{2}\right)^{-1 / 2}$. The quantity $\Sigma_{e}\left(\vec{k}_{1}, \vec{k}_{2}\right)$ is the coherent elastic cross section

$$
\Sigma_{e}\left(\overrightarrow{\mathrm{k}}_{1}, \overrightarrow{\mathrm{k}}_{2}\right)=\left(1+|a|^{2}\right)^{-1} \int\left|F\left(\overrightarrow{\mathrm{k}}_{1}, \overrightarrow{\mathrm{k}}_{2} ; \overrightarrow{\mathrm{n}}\right)\right|^{2} d \Omega \overrightarrow{\mathrm{n}}
$$

The conventional-scattering amplitude satisfies the optical theorem,

$$
\operatorname{Im} f\left(\overrightarrow{\mathrm{k}}_{i} ; \overrightarrow{\mathrm{n}}_{i}\right)=(k / 4 \pi) \sigma(k) \text { for } i=1,2,
$$

where $\sigma(k)$ is the conventional total cross section. The coherent total cross section is the direct sum of the coherent elastic and absorption cross sec- 
tions:

$$
\begin{aligned}
\Sigma\left(\overrightarrow{\mathrm{k}}_{1}, \overrightarrow{\mathrm{k}}_{2}\right) & \equiv \Sigma_{\text {abs }}\left(\overrightarrow{\mathrm{k}}_{1}, \overrightarrow{\mathrm{k}}_{2}\right)+\Sigma_{e}\left(\overrightarrow{\mathrm{k}}_{1}, \overrightarrow{\mathrm{k}}_{2}\right) \\
& =\sigma(k)+\frac{4 \pi}{k\left(1+|a|^{2}\right)} \operatorname{Im}\left[a f\left(\overrightarrow{\mathrm{k}}_{1} ; \overrightarrow{\mathrm{n}}_{2}\right)+a^{*} f\left(\overrightarrow{\mathrm{k}}_{2} ; \overrightarrow{\mathrm{n}}_{1}\right)\right] .
\end{aligned}
$$

By using Eq. (11) and choosing the factor $a$ proper ly, it can be shown that Eq. (12) is equivalent to the following equation:

$$
\begin{aligned}
& -4 \pi f\left(\overrightarrow{\mathrm{k}}_{1} ; \overrightarrow{\mathrm{n}}_{2}\right)+4 \pi f *\left(\overrightarrow{\mathrm{k}}_{2} ; \overrightarrow{\mathrm{n}}_{1}\right)+2 i k \int f *\left(\overrightarrow{\mathrm{k}}_{2} ; \overrightarrow{\mathrm{n}}\right) f\left(\overrightarrow{\mathrm{k}}_{1} ; \overrightarrow{\mathrm{n}}\right) d \Omega_{\mathrm{n}} \\
& +\frac{4 i m}{\hbar^{2}} \int V_{I}(\overrightarrow{\mathrm{r}}) \psi_{2}^{*}(\overrightarrow{\mathrm{r}}) \psi_{1}(\overrightarrow{\mathrm{r}}) d \overrightarrow{\mathrm{r}} \\
& =0 \text {, }
\end{aligned}
$$

where $\psi_{i}(\overrightarrow{\mathrm{r}})(i=1,2)$ is the conventional wave function with the asymptotic form

$$
\psi_{i}(\overrightarrow{\mathrm{r}}) \underset{r \rightarrow \infty}{\longrightarrow} e^{i \overrightarrow{\mathrm{k}}_{i} \cdot \overrightarrow{\mathrm{r}}}+f\left(\overrightarrow{\mathrm{k}}_{i} ; \overrightarrow{\mathrm{n}}\right)\left(e^{i k r} / r\right) .
$$

Equation (13) is often called the generalized optical theorem. ${ }^{2}$ Since the last term has no simple physical interpretation in conventional scattering, the theorem is not useful. ${ }^{2}$ Now coherent scattering has provided new meaning for this theorem.

The conventional-scattering amplitude satisfies the reciprocity theorem ${ }^{2}$ :

$$
f\left(\overrightarrow{\mathrm{k}}_{1} ; \overrightarrow{\mathrm{n}}_{2}\right)=f\left(-\overrightarrow{\mathrm{k}}_{2} ;-\overrightarrow{\mathrm{n}}_{1}\right) .
$$

If the potential $V(\overrightarrow{\mathrm{r}})$ possesses space-inversion symmetry, then

$$
f\left(\overrightarrow{\mathrm{k}}_{1} ; \overrightarrow{\mathrm{n}}_{2}\right)=f\left(-\overrightarrow{\mathrm{k}}_{1} ;-\overrightarrow{\mathrm{n}}_{2}\right) \text {. }
$$

From Eqs. (15) and (16), one can rewrite Eq. (12) as

$$
\Sigma\left(\overrightarrow{\mathrm{k}}_{1}, \overrightarrow{\mathrm{k}}_{2}\right)=\sigma(k)+(8 \pi / k)\left(1+|a|^{2}\right)^{-1} \operatorname{Re}(a) \operatorname{Im} f\left(\overrightarrow{\mathrm{k}}_{1} ; \overrightarrow{\mathrm{n}}_{2}\right) .
$$

This equation is the same as Eq. (3.18) in the previous paper. However, the latter was proved only for a spherical potential.

\footnotetext{
${ }^{1}$ Ming Chiang Li, Phys. Rev. A $\underline{9}, 1635$ (1974).

${ }^{2}$ L. I. Schiff, Quantum Mechanics (McGraw-Hill, New York, 1968).
} 